ROCK-independent, whereas that of the rounded cells is ROCK-dependent. These studies suggest that extracellular protease activity is necessary for cells to maintain an elongated morphology, but that in the absence of this activity, they adapt by using another migration mechanism.

Differences are also observed in the way that cells migrate in slice cultures, compared with $2 \mathrm{D}$ substrates. For example, myogenic precursor cells migrating in situ $u^{4}$ showed directed migration and extended long, highly polarized, persistent protrusions that are not seen in fibroblasts on $2 \mathrm{D}$ substrates. The surrounding environment clearly provides directional cues for migration because once the myogenic precursors moved out of the slices, the large, persistent protrusions were no longer seen and directional migration was lost. Inhibition of Rac signalling by expression of a dominant-negative Rac mutant inhibited migration and abrogated formation of the large, persistent protrusions, whereas expression of constitutively active Rac also inhibited formation of a single protrusion and produced many small protrusions and random migration. Thus, in the in-vivo-like environment of the slice cultures, localized activation of Rac regulates the polarized directed movement of the muscle precursor cells.

The studies described here provide new insights into the function of Rho family signalling in cell migration. The work by Sahai and Marshall ${ }^{7}$ shows a plasticity in cellular migration mechanisms that seems to be driven, at least in part, by changes in the participation of different Rho family members. In their study, Rho is crucial for the migration of rounded cells but not for the migration of the elongated, $\mathrm{PtdInsP}_{3}$-polarized cells. These observations have profound implications for migrationbased therapies and raise many key questions. For example, how does Rho drive the migration of the rounded cells? What induces Rho activity in these cells? Are there other modes of migration and are they the result of differing contributions among Rho family members? Clearly, cells can adapt motility mechanisms to suit their surrounding environment, but we must also now understand what the extrinsic signals and mechanisms are that determine polarity and plasticity during migration.

1. Cukierman, E., Pankov, R., Stevens, D. R. \& Yamada, K. M. Science 294, 1708-1172 (2001).

2. Wolf, K. et al. J. Cell Biol. 160, 267-277 (2003).

3. Hegerfeldt, Y., Tusch, M., Brocker, E. B. \& Friedl, P. Cancer Res. 62, 2125-2130 (2002)

4. Knight, B. et al. Curr. Biol. 10, 576-585 (2000).

5. Srinivasan, S et al. J. Cell Biol. 160, 375-385 (2003).

6. Allen, W. E., Zicha, D., Ridley, A. J. \& Jones, G. E. J. Cell Biol. 141, 1147-1157 (1998).

7. Sahai, E. \& Marshall, C. J. Nature Cell Biol. 5 , 711-719 (2003).

8. Funamoto, S., Meili, R., Lee, S., Parry, L. \& Firtel, R. A Cell 109, 611-623 (2002).

9. Servant, G. et al. Science 287, 1037-1040 (2000).

10. Weiner, O. D. Curr. Opin. Cell Biol. 14, 196-202 (2002).

11. Wang, F. et al. Nature Cell Biol. 4, 513-518 (2002).

12. Nobes, C. D. \& Hall, A. J. Cell Biol. 144, 1235-1244 (1999)

\section{Getting a GPR on spindle asymmetry}

Proper positioning of the spindle during asymmetric cell division ensures correct partitioning of cellular determinants. In Caenorhabditis elegans embryos, displacement of the spindle towards the posterior side of a singlecelled embryo results in asymmetric cleavage. Now, a study by Gotta et al. (Curr. Biol. 13, 1029-1037 (2003)) suggests that GPR-1 and GPR-2, two highly related G-protein regulators from $C$. elegans, may regulate spindle position through spatial asymmetries in G-protein signalling.

Earlier studies have shown the PAR proteins (required for polarization of the embryo) and heterotrimeric $\mathrm{G}$ proteins (consisting of $\alpha, \beta$ and $\gamma$ subunits) regulate spindle asymmetry in the early embryo. Mutation of two Go subunits - GOA1 and GPA16 - resulted in symmetric cell division, even though polarity markers were properly localized, suggesting that G-protein signalling may be involved in coupling polarity signals to spindle position. However, relatively little is known about how GOA-1 or GPA-16 are regulated or how they might govern spindle asymmetry.

In this study, Gotta et al. start by demonstrating that inactivation of GPR1 and GPR-2 results in a symmetric first division. However, they also found that polarity markers such as PAR-2 and PAR-3, which are important for spindle positioning, are correctly localized. This suggests the GPR-1/GPR-2 might function downstream of PAR-2 and PAR-3. Because PAR-2 and PAR-3 are known to regulate pulling forces at the spindle, the authors then assayed for spindle forces. Using inhibitory RNA strategies (RNAi) to block gpr-1/gpr-2 expression, they determined that the forces at both spindle poles in RNAi-treated embryos treated were weak when compared with wild-type embryos. They also found that in single-celled wild-type embryos, GPR-1/GPR-2 was enriched at the pole, where spindle forces are stronger. In addition, the asymmetric distribution of GPR1/GPR-2 was found to be dependent on PAR-2 and PAR-3. These observations begin to provide a framework for understanding how PAR-2 and PAR-3 direct generation of asymmetric forces at the spindle poles.

But how does GPR-1/GPR-2 affect G-protein signalling downstream

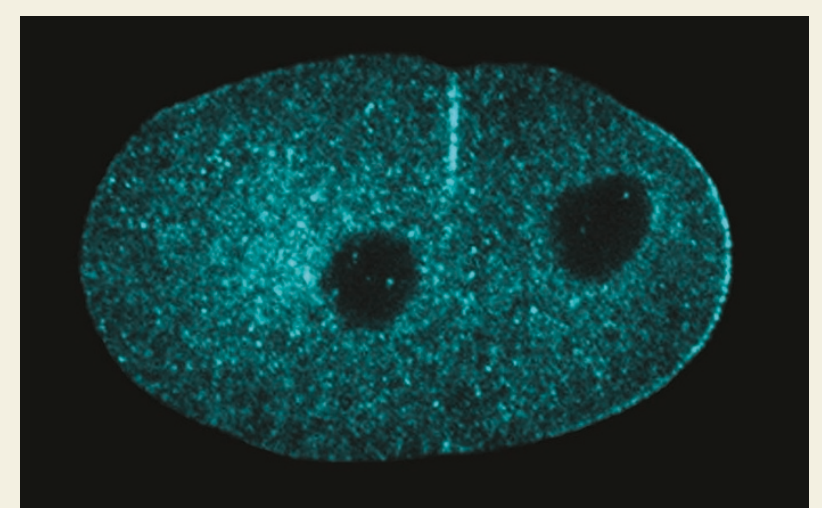

Asymmetric enrichment of GPR-1/GPR-2 at the posterior side of a C. elegans embryo during the first cleavage.

of PAR-2 and PAR-3? The answer may lie in the fact that both GPR-1 and GPR-2 contain a GPR domain. Studies of mammalian and Drosophila melanogaster homologues have shown that the presence of this motif inhibits dissociation of GDP from the G $\alpha$ subunit. Gotta et al. found that GOA-1 binds the GPR motif of both GPR-1 and GPR-2, and that binding specifically inhibited dissociation of GDP from GOA-1.

These findings lead the authors to propose that asymmetric spindle position may be generated by increased signalling through a GOA1-GRP-1/GRP-2 complex at one pole which, in turn, results in stronger pulling forces at this spindle pole. Gotta et al. suggest that formation of this complex might result in activation of targets that directly regulate spindle force. The identification of downstream effectors of the GOA1-GPR-1/GPR-2 complex is an important goal for the future and will undoubtedly provide greater insights into the underlying mechanisms that govern asymmetric spindle position.

SOWMYA SWAMINATHAN 